\title{
A Comparative Study of Various Digital Image Fusion Techniques: A Review
}

\author{
Prabhdeep Kaur \\ Computer Science and Engineering \\ Global Institute Of Management and Emerging \\ Technologies Amritsar
}

\begin{abstract}
The key objective of vision fusion would be to merging information from multiple images of exactly the same view in order to deliver only the useful information. The PCA based ways of vision fusion are more suitable and time-saving in realtime systems using PCA based standards of still images. This paper has centered on the many image fusion techniques. The review has shown that the still much research is needed to improve the image fusion technique further. The IBLPCA based technique has shown quite improved results over the available techniques. This paper ultimately ends up with the suitable future directions.
\end{abstract}

\section{Keywords}

Fusion, PCA, DCT, IBLPCA.

\section{INTRODUCTION}

Image fusion is an essential subject in vision processing. Image fusion is a process of combining the relevant information from a couple of pictures in to a single image where in fact the resulting merged picture may well be more helpful and complete than some of the input pictures. Picture fusion means the combining of two in to a single picture that has the maximum information content without producing details which are nonexistent in a given picture. With rapid development in technology, it's now possible to obtain information from multisource pictures to generate a good quality merged image with spatial and spectral information. Caused by vision fusion is a new vision that retains the most desirable information and characteristics of input vision. Several situations in vision processing require high spatial and high spectral resolve in a single vision. Most of the existing equipment is not capable of providing such records convincingly. In remote sensing and in astronomy, multi sensor merging can be used to reach high spatial and spectral resolution by merging visions from two sensors among that has high spatial resolution and the other one high spectral resolution. The key utilization of vision fusion is merging the grey level high resolution panchromatic vision and the colored low resolution multispectral image. [5] The vision fusion techniques enable the mixture of different information sources. The merged vision may have complementary spatial and spectral resolution features.

When using the vision merging technique, some general requirements should be considered

- The fusion procedure shouldn't discard any information within the source pictures.

- The fusion procedure shouldn't introduce any artifacts or inconsistencies that may distract or mislead a human observer or any subsequent vision processing steps.

- The fusion procedure should be consistent, strong and have, as much as possible, the capacity to tolerate imperfections such as noise or miss registrations.

\author{
Mandeep Kaur \\ Head of department (Information Technology) \\ Global Institute Of Management and Emerging \\ Technologies Amritsar
}
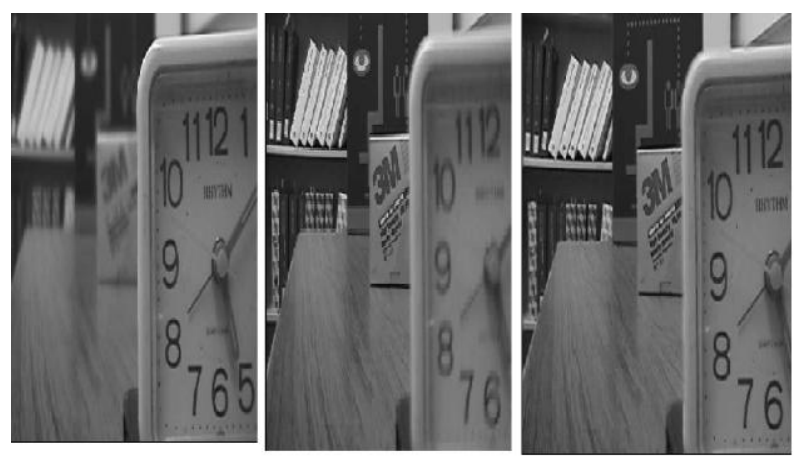

Figure 1:- (a) Input image focused on the foreground (clock region) (b) Input image focused on the background (books region) (c) Fused image [7]

\section{IMAGE FUSION TECHNIQUES}

In the Image Fusion method the required data from the given supply photographs is merged together to make a composite image whose quality is more advanced than the given feedback images. Picture combination methods could be categorized in to two groups' i.e.

- Spatial domain fusion method

- Transform domain fusion

In spatial domain practices, the pixel price of a picture is immediately dealt with. The pixel values are altered to obtain preferred result. Fusion is required in every area where images are required to be examined. For example, medical image analysis, microscopic imaging, analysis of photographs from satellite, remote sensing request, pc vision and battlefield monitoring. An analysis of remote sensing photographs is being performed utilizing the adjustable quality analysis tool. The distinct wavelet convert is among the important method used for fusion. Strategies like these show increased benefits in spatial and spectral quality of the merged image when compared with different spatial types of fusion. [9]

\subsection{Principal Component Analyses (PCA)}

PCA is just a mathematical instrument for change of correlated factors in to uncorrelated factors. For picture classification and picture pressure PCA is used comprehensively. There's involvement of mathematical formula for change of factors which can be called key components. It computes a tight and optimum explanation of the info set. The very first key aspect corresponds to the the maximum amount of difference probable in the info and every subsequent aspect corresponds to the rest of the variance. First key aspect is taken across the direction of optimum variance. The 2 nd key aspect is limited to lay in the subspace at a 90 level angle of the first. Within this Subspace, this aspect items the direction of optimum variance. The next key aspect is taken in the optimum difference direction in the subspace at a 90 level angle to the former two. [9] 


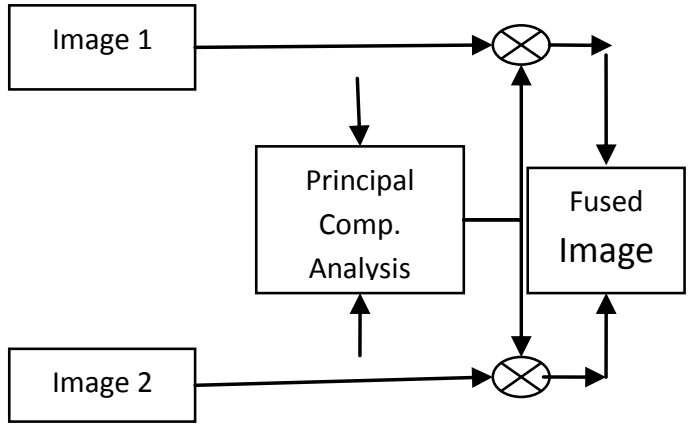

Figure 2: I mage Fusion Process using PCA[3]

\subsection{Discrete Cosine Transform}

It's seen that all the picture fusion methods are extremely complex and consumes long which are tough to be used on real-time applications. The fusion strategies which are used in DCT domain are extremely effective when the feedback photographs are numbered and merged photographs are restored in JPEG standard .For using the JPEG development, an image (in color or gray scales) is divided in to blocks of $8 \times 8$ pixels firstly. The Discrete Cosine Transform (DCT) is a while later used on each block leading to the technology of 64 coefficients that are quantized to decrease their magnitude. The coefficients are then changed into a one-dimensional variety in a crisscross fashion prior to entropy encoding. The compression is obtained in two measures; the initial through quantization and the next through the entropy development process. For reducing the problems undergone in the fusion of real time programs and enhancing the quality of merged picture, DCT fusion strategy is applied. Difference of $8 \times 8$ blocks computed from DCT coefficients is used as a comparison qualification for the experience evaluate. [9]

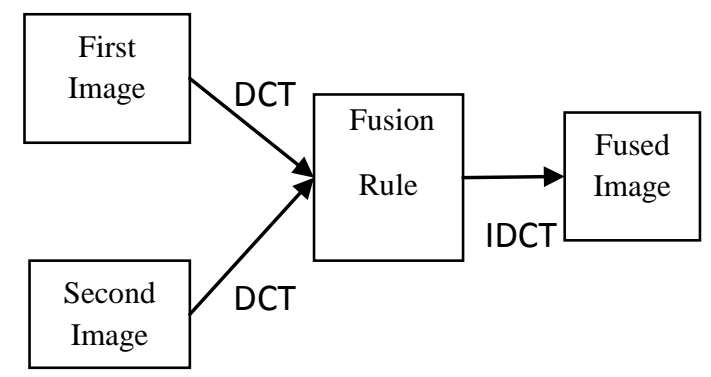

Figure 3: Image Fusion process Using DCT [3]

\subsection{Discrete Wavelet Transform (DWT)}

In discrete wavelet change (DWT) decomposition, the filters are particularly developed to ensure that successive layers of the chart just contain details which are not presently accessible at the preceding levels. The DWT decomposition works on the cascade of special low pass and high-pass filters and a subsampling operation. The components from 2D-DWT are four photographs having measurement add up to half how big is the original picture. [3]

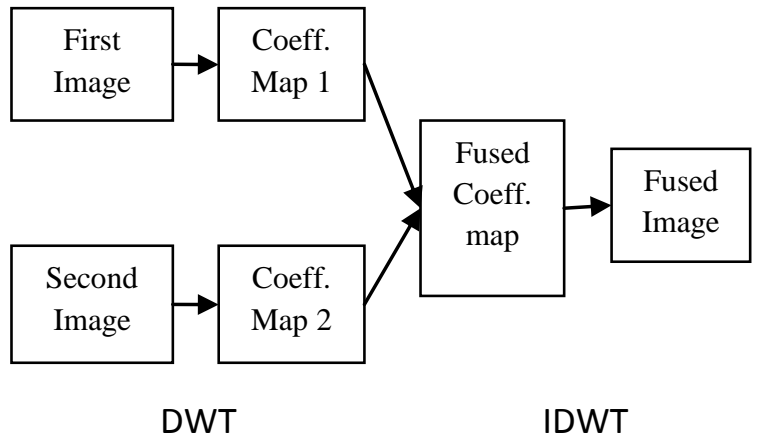

Figure 4: Image Fusion process Using DWT [3]

\section{LITERATURE SURVEY}

Albuquerque et al. (2012) [1] has discussed that vision focus is home closely related to vision quality. In a few images it is extremely hard to get a clear focus in a all regions simultaneously, so an alternate is by using picture fusion to merge images with different focus into one with all the current best focused regions. There are two vision fusion algorithms in the frequency domain that derive from focus- DCT and spatial frequency. The algorithms divide the picture into fixed size of blocks to choose which picture should really be selected to constitute the ultimate result. Ashwini Galande, Ratna Patil (2013) [5] in this study paper, picture blend techniques have been largely labeled in to two types; Pixel stage picture blend and change based picture fusion. Different strategies in each category have been mentioned in detail. Relative analysis is completed on the basis of the limits and benefits of each approach and quantitative analysis is conducted using entropy, UIQI, blend element, blend symmetry and processing time. For analysis function we have regarded the combination of CT and MRI images. It's quite difficult to pick one approach for all applications. But from aesthetic perception, fused photos obtained from unclear inference system give greater result.

Cao et al. (2010) [2] has proposed that multi focus vision fusion handles a stack of imagery that have been acquired with an alternative focus point to get an vision with all the current objects in the scene full focused. Multi focus noisy vision fusion algorithm utilizing the contour let transform has been proposed. Using the captured directional information by the contour let transform, the directional windows are accustomed to determine the fusion weight. Desale et al. (2013) [3] vision Fusion is an activity of combining the related information from a set of images, right into a single vision, where in the resultant fused vision may well be more informative and complete than some of the input images. This paper discusses the Formulation, Process Flow Diagrams and algorithms of PCA (principal Component Analysis), DCT (Discrete Cosine Transform) and DWT (Discrete Wavelet Transform) based vision fusion techniques. The PCA \& DCT are conventional fusion techniques with many drawbacks. Two algorithms centered on DWT are proposed, they're, pixel averaging \& maximum pixel replacement approach. Gupta, R. ; Awasthi, D. (2014) [6] has proposed to fuse CT and MRI images. There have been various standard method used for image fusion produce that produce good spatial result but cause spatial noise. In this paper they represented image fusion technique that may provide better result using Discrete Wave Packet decomposition (DWPT) and optimize result using genetic algorithm (GA) than compare it with Intensity Hue Saturation (IHS) used for image fusion. Performance of purposed fusion technique have been measured by mean, standard deviation, entropy, variance, mutual information, peak 
signal to noise ratio (PSNR) and structure similarity. Haghighat et al. (2010) [7] has studied that the aim of vision fusion would be to merge information from many images of the exact view to be able to deliver only the useful information. The discrete cosine transform based types of vision fusion are considerably better and time-saving in real-time systems using discrete cosine transform based values of unmoving image or video. Hongbo Wu Yanqiu Xing (2010) [17] presented an approach for multi-resolution image fusion of a high-resolution SPOT (Satellite Pour l'Observation de la Terre, SPOT) panchromatic image and a low-resolution Landsat 7 ETM+ multispectral image centered on wavelet transform (WT) combined with filtering in the Fourier domain. Firstly, the images were decomposed with a wavelet coefficients by Mallat algorithm; Secondly, the wavelet transform methods add the wavelet coefficients of the SPOT PAN image to ETM+ image. Finally, the fused image is reconstructed by performing the inverse wavelet transform for obtaining multispectral images of higher spatial resolution. In order to evaluate the caliber of the fused images, three quantitive indicators such as for example Gradients, RMSE, Correlation Coefficients were defined in the paper. Hongyuan Jing (2014) [9] proposed a brand new PCA based versatile picture mix algorithm to blend multi-focus photos with the same aesthetic perspective but different focus. The core of the algorithm is really a new method for evaluating each pixel's covariance matrix with the common covariance matrix. The check results show that the proposed algorithm outperforms state-of the- artwork multi-focus picture mix algorithms. Additionally, the proposed algorithm functions decrease implementation charges, that is suited to the embedded systems. Huaxun Zhang, Xu Cao (2013) [19] in that paper a way of medical picture combination based on wavelet idea was introduced. Medical picture combination have three measures, they are picture processing, picture subscription and picture fusion. Picture processing get across multiresolution traits of wavelet to denoise, picture subscription pass the wavelet evaluation to gain biggish modify stage and get picture side to reach quick and great superposable, picture combination use disassumble picture to different volume subband to save all data to truly have a perfect fusion. Simulation test demonstrated its advantages of only computation, fast superposition and perfect combination in medical picture fusion. It is a direction of medical reacher and clinic iatrology. Jasmeetkaur, Er.Rajdavinder Singh Boparai [8] presented an evaluation of many picture combination methods. The picture combinations have been applied among the common methods in picture processing. Several picture combination methods had been utilized in numerous applications. Picture combination mixes the data from various photos of simple world to get an informative picture which will be right for individual visualisation or more vision processing.Image superiority is a clearly related to picture focus. The key purpose of picture combination is to mix information from numerous photos of the simple world in order to deliver only the valuable information. The distinct cosine turns (DCT) centered methods of picture combination are right and time-saving in real-time techniques applying DCT centered axioms of still image. Lavanya et al. (2011) [10] indicates that the multi sensor vision fusion is the procedure of combining related information from high spatial resolution vision and high spectral resolution vision. This paper proposes a new vision fusion method centered on wavelet combined IHS and PCA transformations for remotely sensed lunar vision data to be able to extract features correctly. Different fusion techniques have now been used previously separately for spatial and spectral quality vision enhancement. Liu et al. (2010) [12] has discussed that vision fusion is an important step for vision mosaic. Fusion algorithms affect the visual effect and quality of the mosaic imagery directly. An adaptive weighted coefficients algorithm for vision fusion has been proposed. Adaptive weighted coefficients algorithm can adjust weighted coefficients adaptively alongside changes of the variety and shape of the overlapping regions. Harris operator can be used to sense feature points. Mumtaz, A. Majid, A.(2008) [13] had studied that there are many Image Fusion techniques based on signal, pixel, feature and symbol level fusion. Genetic Algorithms (GA's) have been used for solving optimization problems. GA can be employed to image fusion where some kind of parameter optimization is required. An existing and three novel image fusion algorithms which use GA's are presented. The experimental results have shown that GA based image fusion algorithms outperform the existing image fusion algorithms. GA based image fusion methods were time consuming, so they cannot be adopted in real time applications, however they can be very helpful in static image fusion applications. Qingping Li, Junping Du (2013) [11] centered on the situation, a region-based multi-focus picture combination system was planned based on the regional spatial frequency (LSF). Firstly, calculate LSF for each pixel of resource pictures, and a segmentation of the common picture was presented to section the source images. From the segmented picture, a distributed area illustration is purchased to label the source images. The identification of essential functions in distributed area illustration, area spatial frequency (RSF), has been used to guide the combination process. The planned system works well in multi-focus picture combination, and it outperforms comparison strategies with regards to aesthetic and objective evaluations. Rong Fan, Qiufen Yang and Guanqun Liu (2014) [4] planned the nonlinear measured multiband combination algorithm which introduced the nonlinear measured value. The simulation effect suggests that the calculated number of the improved image combination algorithm is somewhat decreased, and the real-time efficiency has been improved significantly. In contrast to the traditional Laplacian pyramid combination algorithm, the accuracy of the image combination has been also improved effectively. Varshney, P.K. (2011) [18] investigated the situation of fusion of remote sensing images, e.g., multispectral image fusion, based on MRF models and incorporates the contextual constraints via MRF models to the fusion model. Fusion algorithms underneath the maximum a posteriori criterion are developed to search for solutions. His algorithm has been applicable to both multiscale decomposition (MD)-based image fusion and non-MD-based image fusion. Xiangda Sun, Junping $\mathrm{Du}$ (2013) [16] an improved picture blend methodwasis planned in the paper. Firstly, the source picture has been decomposed in to the lowpass and online band-pass coefficients by non sub sampled contourlet convert (NSCT) for multi-sensor picture blend of the exact same scene. The lowpass portion employs the blend approach to improve the vitality contrast, it completely requires into consideration the vitality contrast of coefficient in addition to the neighboring coefficient characteristics. The worthiness of the coefficient in the online band-pass coefficient relates to its bordering coefficients, and the online band-pass coefficient employs the blend strategy based on context. The planned strategy may not just hold the vitality of the picture, but also can save you the side data of image. Y Phamila , R.Amutha [15] presented a simple and efficient multi-focus image fusion scheme explicitly designed for wireless visual sensor systems equipped with resource constrained, battery powered image sensors employed in surveillance, hazardous environment like battlefields etc. Here the fusion of multi-focus images has been based on higher valued Alternating Current (AC) coefficients calculated in 
Discrete Cosine Transform (DCT) domain. The proposed method overcomes the computation and energy limitation of low power devices and is investigated in terms of image quality and computation energy. Simulations are performed using Atmel Atmega128 processor of Mica 2 mote to measure the resultant energy savings. The experimental results verify the significant efficiency improvement of the proposed method in output quality and energy consumption, when compared with other fusion techniques in DCT domain. Yijian Pei, Jiang Yu (2010) [14] planned an improved distinct wavelet construction based picture combination algorithm, following learning the rules of the distinct wavelet framework. The improvement could be the consideration of the high volume sub band picture place characteristic. The algorithms may efficiently synthesis the helpful data of the each supply picture restored from the numerous sensors. The numerous target picture combination try and medical picture fusion try may validate our planned algorithm has the effectiveness in the picture fusion. Yong Yang (2010) [20] a novel discrete wavelet transform (DWT) based method for medical picture mix is presented. Firstly, the medical photos to be merged are decomposed by the DWT. Secondly, by considering the features of individual aesthetic program (HVS) and the bodily meaning of the wavelet coefficients, new different mix systems are executed on reduced frequency and high frequency artists independently used by window-based reliability verification. Eventually, the merged picture is constructed by the inverse DWT (IDWT) with all the mixed coefficients.

TABLE 1: Comparison Table

\begin{tabular}{|c|c|c|c|c|c|c|}
\hline $\begin{array}{l}\text { REF } \\
\text { NO. }\end{array}$ & $\begin{array}{l}\text { AUTHOR } \\
\text { S }\end{array}$ & YEAR & TECHNIQUES & FEATURES & LIMITATIONS & $\begin{array}{c}\text { POST } \\
\text { PROCESSING }\end{array}$ \\
\hline 1. & Albuqurque & 2012 & $\begin{array}{l}\text { DCT Domain, Spatial } \\
\text { Frequency }\end{array}$ & $\begin{array}{l}\text { Sharp image, Improved } \\
\text { contrasts }\end{array}$ & $\begin{array}{l}\text { It uses some sequential } \\
\text { algorithms, the time spent on it is } \\
\text { higher than other techniques. }\end{array}$ & $\mathrm{NO}$ \\
\hline 2. & $\begin{array}{l}\text { Cao, Jian- } \\
\text { Zhong }\end{array}$ & 2010 & $\begin{array}{l}\text { Contourlet transform } \\
\text { directional windows }\end{array}$ & $\begin{array}{l}\text { Captures directional } \\
\text { information of natural images, } \\
\text { Successfully used in } \\
\text { compression }\end{array}$ & $\begin{array}{l}\text { It needs to find the image fusion } \\
\text { techniques (which can be } \\
\text { combined) for better results. }\end{array}$ & $\mathrm{NO}$ \\
\hline 3. & $\begin{array}{l}\text { Desale, } \\
\text { V.verma }\end{array}$ & 2013 & $\begin{array}{l}\text { PCA, DCT, DWT } \\
\text { based image } \\
\text { techniques }\end{array}$ & $\begin{array}{c}\text { Superior quality of image is } \\
\text { available }\end{array}$ & $\begin{array}{l}\text { Greater computational } \\
\text { requirements. }\end{array}$ & $\mathrm{NO}$ \\
\hline 4. & Fan, Rong & 2014 & $\begin{array}{l}\text { Non linear weighted, } \\
\text { Non subsampled } \\
\text { contourlet transform }\end{array}$ & Enhanced image quality & High computational complexities. & YES \\
\hline 6. & $\begin{array}{l}\text { Gupta, } \\
\text { Richa }\end{array}$ & 2014 & Genectic Algorithm & $\begin{array}{l}\text { Fused image obtained after } \\
\text { fusion has characteristics of } \\
\text { each image }\end{array}$ & Vast research domain. & NO \\
\hline 7. & $\begin{array}{l}\text { Haghighat, } \\
\text { Mohamma } \\
\text { d }\end{array}$ & 2010 & DCT domain & $\begin{array}{l}\text { Efficiency improvement both } \\
\text { in quality and complexity } \\
\text { reduction }\end{array}$ & $\begin{array}{l}\text { Weak on boundaries b/w focused } \\
\text { and out of focused areas. }\end{array}$ & $\mathrm{NO}$ \\
\hline 8. & $\begin{array}{l}\text { Jasmeet } \\
\text { Kaur }\end{array}$ & 2013 & $\begin{array}{l}\text { DCT(Discrete Cosine } \\
\text { Transform) }\end{array}$ & $\begin{array}{l}\text { Extract valuable information } \\
\text { from source images without } \\
\text { introduction of artifacts }\end{array}$ & $\begin{array}{l}\text { Fusion quality can be further } \\
\text { improved, Works only for still } \\
\text { images/videos. }\end{array}$ & $\mathrm{NO}$ \\
\hline 9. & $\begin{array}{c}\text { Jing, } \\
\text { Hongyuan }\end{array}$ & 2014 & $\begin{array}{l}\text { PCA, Multifocus } \\
\text { image fusion }\end{array}$ & $\begin{array}{c}\text { Removes redundant } \\
\text { information from input image } \\
\text { and reconstruct output image }\end{array}$ & $\begin{array}{l}\text { PCA focus short when it comes to } \\
\text { determining the true impact of } \\
\text { principle components, Depth of } \\
\text { focus is limitation of multifocus } \\
\text { image fusion. }\end{array}$ & $\mathrm{NO}$ \\
\hline 11. & $\begin{array}{c}\mathrm{Li}, \\
\text { Qingping }\end{array}$ & 2013 & $\begin{array}{l}\text { Local Spatial } \\
\text { Frequency }\end{array}$ & $\begin{array}{l}\text { Has better performances in } \\
\text { terms of both visual quality } \\
\text { and objective evaluation }\end{array}$ & $\begin{array}{l}\text { The detector pixel spacing defines } \\
\text { its limitations. }\end{array}$ & $\mathrm{NO}$ \\
\hline 12. & $\begin{array}{l}\text { Liu, } \\
\text { Haifeng }\end{array}$ & 2010 & $\begin{array}{l}\text { Adaptive Weighted } \\
\text { Coefficients }\end{array}$ & $\begin{array}{l}\text { Shows better fusion results, } \\
\text { Mosaic images are smooth } \\
\text { and natural }\end{array}$ & Feature points need to be sensed. & $\mathrm{NO}$ \\
\hline
\end{tabular}




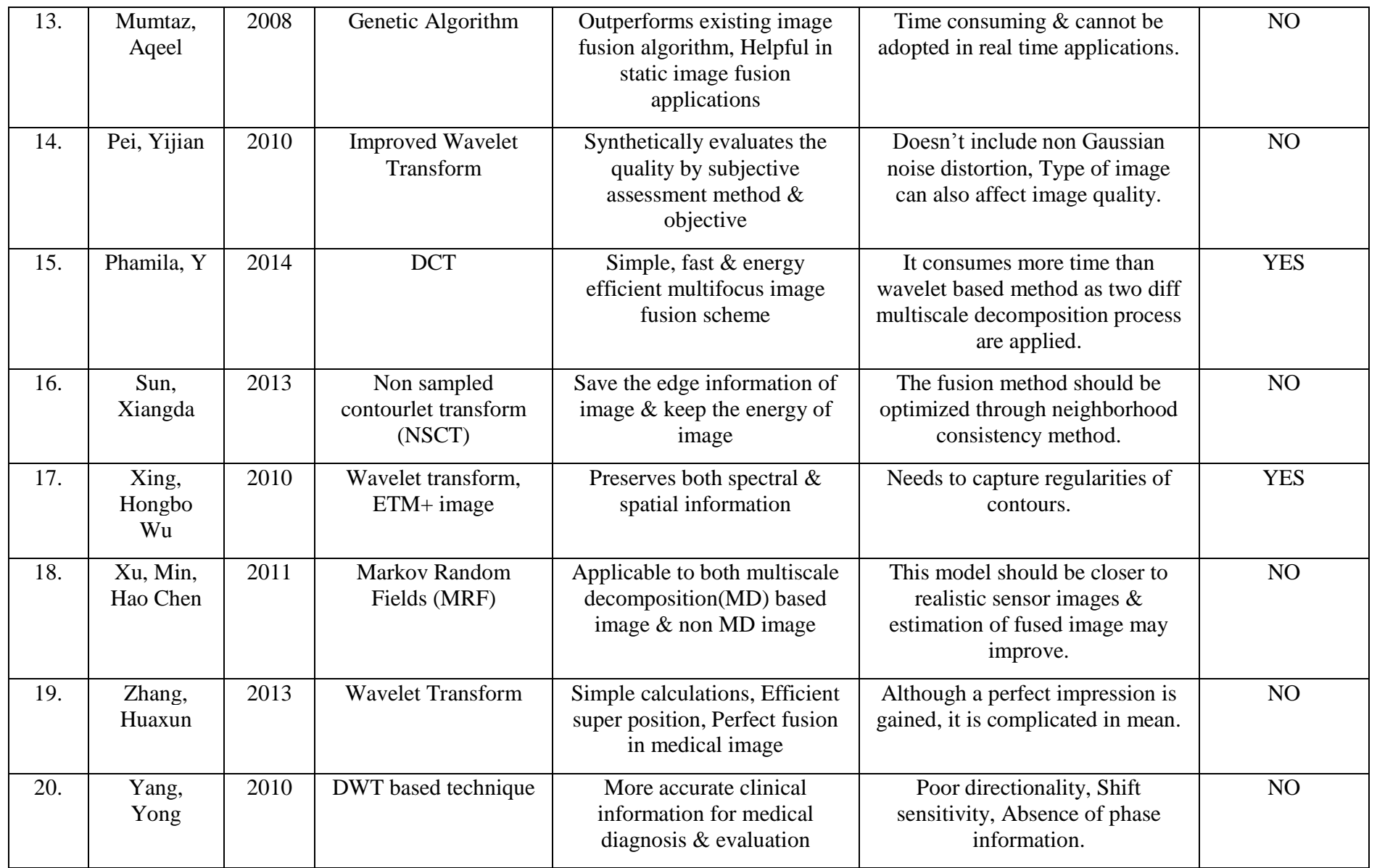

\section{GAPS IN LITERATURE}

By conducting the review it has been discovered that the all the existing literature has neglected one or more of the following.

1. As all the existing methods are in relation to transform domain therefore it might results in a few color artifacts that might decrease the performance of the transform based vision fusion methods.

2. It is also discovered that the situation of the uneven illuminate has been neglected in probably the most of existing work on fusion.

3. Most of the existing work has focused on gray scale images very little work is performed for color images.

\section{CONCLUSION AND FUTURE WORK}

By conducting the review it's been unearthed that the all of the existing literature has neglected many issues. As all of the existing methods are based upon transform domain therefore it may results in certain color artifacts which may decrease the performance of the transform based vision fusion methods. It is also unearthed that the problem of the uneven illuminate has been neglected in the absolute most of existing focus on fusion. A lot of the existing work has centered on gray scale images little work is performed for color images. In foreseeable future we shall propose a brand new technique that'll integrate the larger valued AC coefficients calculated in PCA domain based fusion with illuminate normalization with adaptive histogram equalization to cut back along with artifacts which is introduced as a result of transform domain method i.e. PCA. The fusion process may degrades the sharpness of the edges in the digital images so to overcome this problem edge preserving smoothing is likely to be integrated with proposed algorithm to boost the outcome further.

\section{REFERENCES}

[1] Albuquerque, Hugo R., Tsang Ing Ren, and George DC Cavalcanti 2012 "Image Fusion Combining Frequency Domain Techniques Based on Focus." Tools with Artificial Intelligence (ICTAI), 2012 IEEE 24th International Conference on. Vol. 1. IEEE.

[2] Cao, Jian-zhong, Zuo-feng Zhou, Hao Wang, and Wei-hua Liu 2010 "Multifocus Noisy Image Fusion Algorithm Using the Contourlet Transform." In Multimedia Technology (ICMT), 2010 International Conference on, pp. 1-4. IEEE.

[3] Desale, Rajenda Pandit, and Sarita V. Verma 2013 "Study and analysis of PCA, DCT \& DWT based image fusion techniques." In Signal Processing Image Processing \& Pattern Recognition (ICSIPR), 2013 International Conference on, pp. 66-69. IEEE.

[4] Fan, Rong, Qiufen Yang, and Guanqun Liu 2014 "Nonlinear weighted multiband fusion image algorithm." In Electronics, Computer and Applications, 2014 IEEE Workshop on, pp. 449-452. IEEE.

[5] Galande, Ashwini, and Ratna Patil 2013 "The art of medical image fusion: A survey." In Advances in Computing, Communications and Informatics (ICACCI), 2013 International Conference on, pp. 400-405. IEEE.

[6] Gupta, Richa, and Deepak Awasthi 2014"Wave-packet image fusion technique based on genetic algorithm." In Confluence The Next Generation Information 
Technology Summit (Confluence), 2014 5th International Conference-, pp. 280-285. IEEE.

[7] Haghighat, Mohammad Bagher Akbari, Ali Aghagolzadeh, and Hadi Seyedarabi 2010 "Real-time fusion of multi-focus images for visual sensor networks." In Machine Vision and Image Processing (MVIP), 2010 6th Iranian, pp. 1-6. IEEE.

[8] Jasmeetkaur, Er, and Rajdavinder Singh Boparai 2013 "AN EVALUATION ON DIFFERENT IMAGE FUSION TECHNIQUES."vol.4.

[9] Jing, Hongyuan, and Tanya Vladimirova 2014 "Novel PCA based pixel-level multi-focus image fusion algorithm." In Adaptive Hardware and Systems (AHS), 2014 NASA/ESA Conference on, pp. 135-142. IEEE.

[10] Lavanya, A., K. Vani, S. Sanjeevi, and R. S. Kumar 2011 "Image fusion of the multi-sensor lunar image data using wavelet combined transformation." In Recent Trends in Information Technology (ICRTIT), 2011 International Conference on, pp. 920-925. IEEE.

[11] Li, Qingping, Junping Du, Fuzhao Song, Chao Wang, Honggang Liu, and Cheng Lu 2013 "Region-based multifocus image fusion using the local spatial frequency." In Control and Decision Conference (CCDC), 2013 25th Chinese, pp. 3792-3796. IEEE.

[12] Liu, Haifeng, Mike Deng, Chuangbai Xiao, and Xiao Xu 2010 "Image fusion algorithm based on adaptive weighted coefficients." In Signal Processing (ICSP), 2010 IEEE 10th International Conference on, pp. 748-751. IEEE.

[13] Mumtaz, Aqeel, and Abdul Majid 2008 "Genetic algorithms and its application to image fusion."
In Emerging Technologies, 2008. ICET 2008. 4th International Conference on, pp. 6-10. IEEE.

[14] Pei, Yijian, Huayu Zhou, Jiang Yu, and Guanghui Cai 2010 "The improved wavelet transform based image fusion algorithm and the quality assessment." In Image and Signal Processing (CISP), 2010 3rd International Congress on, vol. 1, pp. 219-223. IEEE.

[15] Phamila, Y., and R. Amutha 2014 "Discrete Cosine Transform based fusion of multi-focus images for visual sensor networks." Signal Processing 95 : 161-170.

[16] Sun, Xiangda, Junping Du, Qingping Li, Xiaoyong Li, Liang Xu, and Yawen Li 2013 "Improved energy contrast image fusion based on nonsubsampled contourlet transform." In Industrial Electronics and Applications (ICIEA), 2013 8th IEEE Conference on, pp. 1610-1613. IEEE

[17] Xing, Hongbo Wu Yanqiu 2010 "pixel-based image fusion using wavelet transform for spot and etm+ image." Progress in Informatics.

[18] Xu, Min, Hao Chen, and Pramod K. Varshney 2011 "An image fusion approach based on Markov random fields." Geoscience and Remote Sensing, IEEE Transactions on 49, no. $12: 5116-5127$.

[19] Zhang, Huaxun, and Xu Cao 2013 "A Way of Image Fusion Based on Wavelet Transform." In Mobile Ad-hoc and Sensor Networks (MSN), 2013 IEEE Ninth International Conference on, pp. 498-501. IEEE.

[20] Yang, Yong 2010 "Multimodal medical image fusion through a new DWT based technique." In Bioinformatics and Biomedical Engineering (iCBBE), 2010 4th International Conference on, pp. 1-4. IEEE. 